\title{
Motor Performance and Physical Activity as Predictors of Prospective Falls in Community- Dwelling Older Adults by Frailty Level: Application of Wearable Technology
}

\author{
M. Jane Mohler ${ }^{a-c}$ Christopher S. Wendel ${ }^{a, b}$ Ruth E. Taylor-Piliae ${ }^{a, c, d}$ \\ Nima Toosizadeh $^{\mathrm{a}, \mathrm{c}}$ Bijan Najafia, ${ }^{\mathrm{a}, \mathrm{e}}$ \\ ${ }^{a}$ Arizona Center on Aging, College of Medicine, ${ }^{b}$ Section of Geriatrics and Gerontology, Department of Medicine, \\ College of Medicine, ' Interdisciplinary Consortium on Advanced Motion Performance (iCAMP), Department \\ of Surgery, and ${ }^{\mathrm{d} C o l l e g e}$ of Nursing, University of Arizona, Tucson, Ariz., and ${ }^{\mathrm{e}}$ Interdisciplinary Consortium on \\ Advanced Motion Performance (iCAMP), Division of Vascular Surgery and Endovascular Therapy, Michael E. \\ DeBakey Department of Surgery, Baylor College of Medicine, Houston, Tex., USA
}

\section{Key Words}

Frailty · Falls · Wearable sensors · Monitoring · Physical function $\cdot$ Physical activity $\cdot$ Balance $\cdot$ Gait

\begin{abstract}
Background: Few studies of the association between prospective falls and sensor-based measures of motor performance and physical activity (PA) have evaluated subgroups of frailty status separately. Objective: To evaluate wearable sensor-based measures of gait, balance, and PA that are predictive of future falls in community-dwelling older adults. Methods: The Arizona Frailty Cohort Study in Tucson, Arizona, followed community-dwelling adults aged 65 years and over (without baseline cognitive deficit, severe movement disorders, or recent stroke) for falls over 6 months. Baseline measures included Fried frailty criteria: in-home and sensor-based gait (normal and fast walk), balance (bipedal eyes open and eyes closed), and spontaneous daily PA over $48 \mathrm{~h}$, measured using validated wearable technologies. Results: Of the 119 participants (36\% non-frail, 48\% pre-frail,
\end{abstract}

and $16 \%$ frail), 48 reported one or more fall ( $47 \%$ of non-frail, $33 \%$ of pre-frail, and $47 \%$ of frail). Although balance deficit and PA were independent fall predictors in pre-frail and frail groups, they were not sensitive to predict prospective falls in the non-frail group. Even though gait performance deteriorated as frailty increased, gait was not a predictor of prospective falls when participants were stratified based on frailty status. In pre-frail and frail participants combined, center of mass sway [odds ratio $(O R)=5.9,95 \%$ confidence interval $(\mathrm{Cl})$ 2.6-13.7], PA mean walking bout duration $(\mathrm{OR}=$ $1.1,95 \% \mathrm{Cl} 1.0-1.2)$, PA mean standing bout duration (OR = $0.94,95 \% \mathrm{Cl} 0.91-0.99)$, and a fall in previous 6 months ( $\mathrm{OR}=7.3,95 \% \mathrm{Cl} 1.5-36.4$ ) were independent predictors of prospective falls (area under the curve: 0.882). Conclusion: This study suggests that independent predictors of falls are dependent on frailty status. Among sensor-derived parameters, balance deficit, longer typical walking episodes, and shorter typical standing episodes were the most sensitive predictors of prospective falls in the combined pre-frail and frail sample. Gait deficit was not a sensitive fall predictor in the context of frailty status.

(c) 2016 S. Karger AG, Basel

\section{KARGER}

(c) 2016 S. Karger AG, Basel

0304-324X/16/0626-0654\$39.50/0

E-Mail karger@karger.com

www.karger.com/ger
M. Jane Mohler, NP-C, MPH, PhD

Arizona Center on Aging, College of Medicine, University of Arizona

1821 E. Elm Street

Tucson, AZ 85719 (USA)

E-Mail jmohler@aging.arizona.edu 


\section{Introduction}

Falling is a significant cause of injuries, loss of mobility, institutionalization, and mortality in older adults [1]. In community-dwelling adults, 35-45\% of those over 65 years old report a fall every year, which rises to $50 \%$ in those over 80 years old [2]. The high susceptibility to injury in the elderly, for example due to clinical diseases such as osteoporosis or impaired protective reflexes that tend to slow with age, increases the danger of even a relatively mild fall [3]. Furthermore, recovery from fall injury is often delayed in older persons, which leads to deconditioning and further increased fall risk [4]. Many elders who fall experience post-fall syndrome, marked by anxiety, fear of falling, and overcautious walking, which can contribute to further deconditioning, weakness and reduced quality of life [5].

Evaluation of the risk of falling is a necessary step towards the provision of preventive measures for individuals deemed to have a high risk of falling. In addition, systematic screening for fall risk in frail elders would help identify those at 'high risk of falls' and in need of specialized geriatric services such as physical rehabilitation. The risk of falling is currently evaluated using questionnaires and short clinical tests of function (e.g., assessments of posture and gait, independence in daily life, cognition, and vision) which have limited precision, and record a single snapshot in time not indicative of activities of daily living. Development of objective and practical tools to track risk of falling in clinic and in home could provide valuable information to reduce fall risk by implementing personalized interventions to address specific motor performance deficit(s). Wearable electro-mechanical sensor technology has provided a new approach for objective measurement of motor performance (including balance and gait) and spontaneous physical activity (PA) under natural conditions [6]. Because wearable sensors are portable and relatively low-cost, they are suitable for realworld fall risk assessment in the complex home and community environments, where most falls occur $[7,8]$. Although wearable technologies have been widely used for assessing risk of falling based on measuring moving ability and motor performance, to date, there has been limited work predicting prospective falls based on monitoring motor performance and daily PA with wearable sensors $[9,10]$. Other reports on PA and fall risk have used nonlinear frequency analysis to extract quality parameters (gait characteristics) from free living activity data, although not all included prospective fall outcomes [1113]. Additionally, it is unclear whether the same model for prediction of prospective falls based on motor performance and PA could be generalized within older adults with different frailty status. In other words, the likelihood of falls may depend on activity level and frailty status of older adults, and thus it stands to reason that fall risk based on motor performance and PA should be assessed in the context of frailty status.

The association between PA and falls has been debated in the previous literature. Klenk et al. [14] did not observe an association between falls per person-year and average of daily PA among 1,214 community-dwelling older people. Interestingly, they observed the highest rate of falls in less active persons with low walking speed. Considering that speed is an important indicator of frailty, this may suggest that the association between PA and falls would be clarified if results are stratified based on frailty status. Frailty is a geriatric syndrome resulting from age-related cumulative decline across multiple physiologic systems, impaired homeostatic reserve, and reduced capacity to resist stress [15]. Frailty increases vulnerability towards adverse health outcomes including falls, hospitalization, institutionalization, and mortality [15]. A recent systematic review estimated that $10.7 \%$ of community-dwelling adults aged 65 and older are frail and $41.6 \%$ are pre-frail [16]. The Arizona Frailty Cohort Study was undertaken to identify relevant sensor-based markers of motor performance and PA that are useful for home-based frailty screening [17]. The study employed one of the most commonly accepted operational definitions of frailty syndrome proposed by Fried et al. [15], which is based on weight loss, weakness, exhaustion, slowness, and low energy expenditure. The aim of this report was to evaluate sensor-based measures of gait, balance, and PA that are predictive of prospective falls in a cohort of communitydwelling adults aged 65 and older that is stratified based on frailty status. We hypothesized that sensor-based baseline assessments can predict prospective fall events in older adults, independent of participant characteristics and retrospective fall history.

\section{Methods}

Study Participants

Reported data were abstracted from the NIH-funded Arizona Frailty Cohort Study, an observational descriptive study of individuals 65 years or older conducted in Tucson, Arizona [17]. Participants were recruited from primary, secondary and tertiary health care settings, community providers, assisted living facilities, retirement homes, and aging service organizations. Adults aged $\geq 65$ years and without gait or mobility disorders (Parkinson's disease, multiple sclerosis, or recent stroke) who reported being able 
to walk $\geq 9.14 \mathrm{~m}$ (30 feet) with or without an assistive device were eligible to be screened for study entry. Exclusion criteria included a Mini-Mental State Examination [18] score $\leq 23$, terminal illness, or unwillingness to participate. Eligible subjects signed a written informed consent form, approved by the University of Arizona institutional review board. In-home assessments were completed between September 2012 and July 2014 by trained clinical research coordinators.

\section{Measures}

Participant Characteristics

A team of 2 trained clinical coordinators visited patients within their home or assisted living setting for collecting data, including gait, balance, and daily PA measurement. Measures included selfreported history of falls, use of assistive device (cane or walker), and number of prescriptions. Demographic and health history data (age, sex, race/ethnicity, and daily medication count) were gathered through self-report. Height was taken on-site with a measuring tape between the floor and a pen held perpendicularly to a wall at the top of the participant's head, and weight was measured using a bathroom scale (Ozeri Touch II, Ozeri ${ }^{\mathrm{TM}}$, Calif., USA) which provided weight, body fat percentage, and muscle percentage. Additionally, participants answered questionnaires to assess tiredness when performing mobility-related tasks on the Mobility-Tiredness Scale [19], depression using the Center for Epidemiologic Studies Depression Scale [20], and independence as reflected by performance in activities of daily living from the Barthel Activities of Daily Living Index [21]. Individuals were also asked to assess their concerns about falling using the Falls Efficacy Scale-International (FES-I), which poses 16 questions regarding the level of fear of falling across various situations [22]. A Timed Up and Go (TUG) test was administered, in which coordinators measured required time for the participant to rise from a chair, walk $3 \mathrm{~m}$, turn around, walk back to the chair, and sit down, at participant's self-selected speed [23].

\section{Frailty Assessment}

Frailty was assessed using the five components specified in the Fried Frailty Phenotype criteria [15], including self-reported unintentional weight loss of 10 pounds or more in the previous year; weakness, based on grip strength test, stratified by gender and body mass index; slow walking speed, stratified by gender and height; self-reported exhaustion, and low energy expenditure, stratified by gender [24], based on the short version Minnesota Leisure Time Activity questionnaire [25]. Following this algorithm, subjects were categorized as non-frail if they met none of the criteria, pre-frail if they met one or two criteria, and frail if they met three or more criteria. This scale has exhibited high validity and has become a gold standard for classifying frailty in adults over the age of 65 [17].

\section{Prospective Falls Ascertainment}

Prospective fall incidence (falls occurring in the 6 months after the initial baseline study visit) were recorded and reported by participants. A fall was considered to be an unexpected event in which the participants unintentionally come to rest on the ground, floor, or a lower level $[26,27]$. Participants were instructed to record all falls prospectively on a provided 6-month weekly fall diary log (date, time, activity prior to the fall, injury symptoms, and need for medical attention), and additionally report all falls by telephone to the study coordination office. A telephone interview after each reported call confirmed details of falls and injuries and resolved any missing data. Fall logs were collected in person at the 6-month follow-up visit. Each participant was dichotomously categorized as a non-faller or a faller (at least one fall during the 6-month follow-up period).

\section{Sensor-Derived Balance, Gait, and PA Parameters}

Balance and gait trials were performed using a validated wearable technology of five small inertial sensors (tri-axial accelerometer and gyroscope) attached to the shins above ankles, thighs above knees, and lower back close to the sacrum (LEGSys ${ }^{\mathrm{TM}}$; BioSensics, Cambridge, Mass., USA) [28, 29]. Balance during quiet standing was measured in two trials of $15 \mathrm{~s}$, one with eyes open with no visual target specified, and one with eyes closed. For balance tests, participants were asked to stand silently and erectly with their arms crossed across their chest, and their feet as close together as possible without touching. The balance software (BalanSens ${ }^{\mathrm{TM}}$, BioSensics) analysis included sway of hip, ankle, and center of mass (COM) extracted from the sensors attached on the right shin and the lower back [30,31]. The ankle, hip, and COM sways are the product of medial-lateral and anterior-posterior sways for each parameter.

Gait assessment was conducted as participants walked a distance of $4.57 \mathrm{~m}$ (15 feet) in their home at a self-selected speed. The gait software (LEGSys ${ }^{\mathrm{TM}}$ ) analysis included gait speed, stride time, stride length, double support (as percentage of stride time), and gait variability (coefficient of variation of stride velocity), based on validated algorithms and data extracted from all five sensors [29, 32]. Participants who reported regular daily use of assistive devices (canes or walkers) used their device for the gait assessments.

PA, including posture durations (i.e., sitting, standing, walking, and lying), postural transitions, and locomotion outcomes (i.e., number of walking bouts, steps per day; distribution of steps per walking bout, and cadence), was monitored over a 48-hour period using a validated triaxial accelerometer wearable technology device (PAMSys ${ }^{\mathrm{TM}}$, BioSensics), which was inserted into a T-shirt with a device pocket located at the sternum. Participants were advised to wear this shirt at all times, except while showering. The device was able to identify postural transitions and movements such as walking, standing, sitting, or lying, which are described in detail elsewhere [32].

\section{Statistical Analysis}

To compare demographic and clinical characteristics between fallers and non-fallers, we used Student's t tests for continuous variables, $\chi^{2}$ tests for categorical variables (Fisher's exact test if any cell had less than 5 participants), and the Cochran-Armitage (score) test for ordinal variables. The Mann-Whitney U test was used to compare sensor-based measures of gait, balance, and PA between non-fallers and fallers due to non-normal distribution of many of these variables. We compared participant characteristics and wearable sensor-based measures between fallers and non-fallers in a separate column for each frailty category (frail, pre-frail, and nonfrail) due to observed significant interactions between frailty category and certain characteristics in predicting faller status. We calculated means, standard deviations (or frequencies of categorical variables), $\mathrm{p}$ values, and Cohen's d effect sizes for the sensor-based parameter differences. We used logistic regression to examine the association between frailty and the risk of being a faller (at least one fall) or a recurrent faller (at least two falls), using two indicator variables of pre-frail and frail, referenced to non-frail. 
We used multiple logistic regression to examine the relationship of participant characteristics and wearable sensor-based measures with the risk of falling. Due to significant interactions observed between several sensor-based parameters and frailty status, we constructed separate models for frail/pre-frail combined and for nonfrail. Most notably, for the COM sway there was a significant interaction $(\mathrm{p}=0.031)$ indicating that frail/pre-frail had a 5 -fold increased risk of falling with every square centimeter increase in COM sway, whereas non-frail subjects had no increased risk, adjusted for history of falls in previous 6 months. The rationale for combining frail and pre-frail groups was: (1) the frailty sample size by itself lacked sufficient power to detect associations if they existed; (2) effects for a given predictor variable were almost always in the same direction (and when not, the effect size was extremely close to zero), and (3) the interactions observed were strongest and more often statistically significant when evaluated with an indicator of frail/pre-frail combined versus non-frail. However, after regression models were constructed in pre-frail and frail combined, we inspected the final model in pre-frail and frail separately to judge whether combining the subgroups may have biased results.

We first used univariate logistic regression with fallers versus non-fallers as the dependent variable to test the relationship with candidate variables. Candidate variables were selected if the $p$ value was $<0.20$ in comparisons between fallers and non-fallers in the pre-frail or frail groups by the Mann-Whitney $U$ test or $\chi^{2}$ test. All independent variables in regression were continuous, except fall history in the previous 6 months and use of assistive devices, which were dichotomous. Odds ratios (ORs), 95\% confidence intervals (CIs), standardized ORs (raw coefficient times standard deviation of independent variable) and p values are reported.

Next, sequential multiple logistic models were constructed by adding blocks of related independent variables for which the $\mathrm{p}$ value was $<0.20$ in the univariate logistic regression, as well as retaining any independent significant predictor $(\mathrm{p}<0.05)$ from the previous multiple logistic model. When two or more parameters within a block showed collinearity $(r>0.90)$, those with higher univariate $\mathrm{p}$ values were excluded. The sequential models were constructed as follows: model 1, balance parameters; model 2, gait parameters; model 3, PA parameters, and model 4, subject characteristics. Finally, model 5 retained all independent significant predictors $(\mathrm{p}<$ 0.05 ) from model 4. For each multiple logistic regression model, we calculated: pseudo $\mathrm{R}^{2}$, a measure of model goodness of fit; area under the curve (AUC) from receiver operating characteristic analysis, a measure of model ability to discriminate between fallers and nonfallers, and Akaike information criteria (AIC), a relative measure of model goodness of fit and efficiency allowing comparison of models that are not nested (all terms of the smaller model occur in a larger model). Since AIC comparisons require the same sample across all models, it was derived from models on the subset of 55 participants with no missing block variables. Statistical analysis was performed using Stata version 14.0 (Statacorp, College Station, Tex., USA).

\section{Results}

\section{Demographic and Clinical Characteristics}

The Arizona Frailty Cohort Study included 128 subjects, of which 9 dropped out after baseline assessment.
All 119 who completed the study provided prospective fall diaries and telephone confirmation; although, by the time of the 6 -month visit, $5 \%$ had lost diaries, which were recreated based on telephone call records and prompting by the coordinator. The completed sample included 43 (36\%) non-frail, 57 (48\%) pre-frail, and 19 (16\%) frail participants. Almost $50 \%$ of both non-frail $(n=20,47 \%)$ and frail $(n=9,47 \%)$ participants experienced at least one fall during the 6 -month follow-up, but only $33 \%$ of prefrail $(n=19)$ participants did so. The risk of being a faller in the follow-up was not significantly associated with being frail (OR 1.00, 95\% CI 0.35-3.1, p = 0.95) or pre-frail $(\mathrm{OR}=0.58,95 \% \mathrm{CI} 0.25-1.29, \mathrm{p}=0.18)$. The number of participants with two or more falls (recurrent faller) was $13(30 \%)$ non-frail, 5 (9\%) pre-frail, and 3 (16\%) frail. The risk of being a recurrent faller in the follow-up was not significantly associated with being frail (OR $0.43,95 \%$ CI $0.11-1.7, p=0.24)$, but was significantly reduced in the pre-frail group $(\mathrm{OR}=0.32,95 \% \mathrm{CI} 0.12-0.90, \mathrm{p}=0.03)$. Fear of falling (FES-I) increased significantly with increasing frailty status $(20.8 \pm 4.2$ non-frail, $28.9 \pm 10.8$ pre-frail, and $41.4 \pm 12.8$ frail, $\mathrm{p}<0.001$ between each category, Bonferroni adjusted).

Table 1 shows participant demographic and clinical characteristics, comparing fallers with non-fallers separately for each frailty category. Age increased across frailty categories, but was not significantly different by faller status within frailty categories. Fallers in the pre-frail group were significantly more likely to have reported a fall in the previous 6 months compared to non-fallers ( $\mathrm{p}=0.001$ ), but this commonly reported association was not significant in the non-frail $(\mathrm{p}=0.08)$ or frail groups $(\mathrm{p}=0.35)$. Fallers in the pre-frail group were significantly more likely to use an assistive device (cane or walker) compared to non-fallers $(\mathrm{p}=0.02)$, but the differences were not significant in the non-frail ( $p=0.32)$ or frail groups $(p=0.35)$. However, all of the non-fallers who used assistive devices in the frail group used a walker, which may indicate a different fall risk from a cane. In the frailty group, fallers had a 5.3-point $(43 \%)$ higher mean depression score that was not statistically significant $(p=0.09)$. Notably, the Timed Up and Go performance test did not discriminate between fallers and non-fallers, either as a continuous measure or as a dichotomous measure of $\geq 13.5 \mathrm{~s}$. No other significant associations with faller status were observed.

\section{Balance}

Table 2 shows wearable sensor-based balance parameters, comparing fallers with non-fallers separately for each frailty category. In the pre-frail group during eyes 
Table 1. Participant characteristics by incident faller status

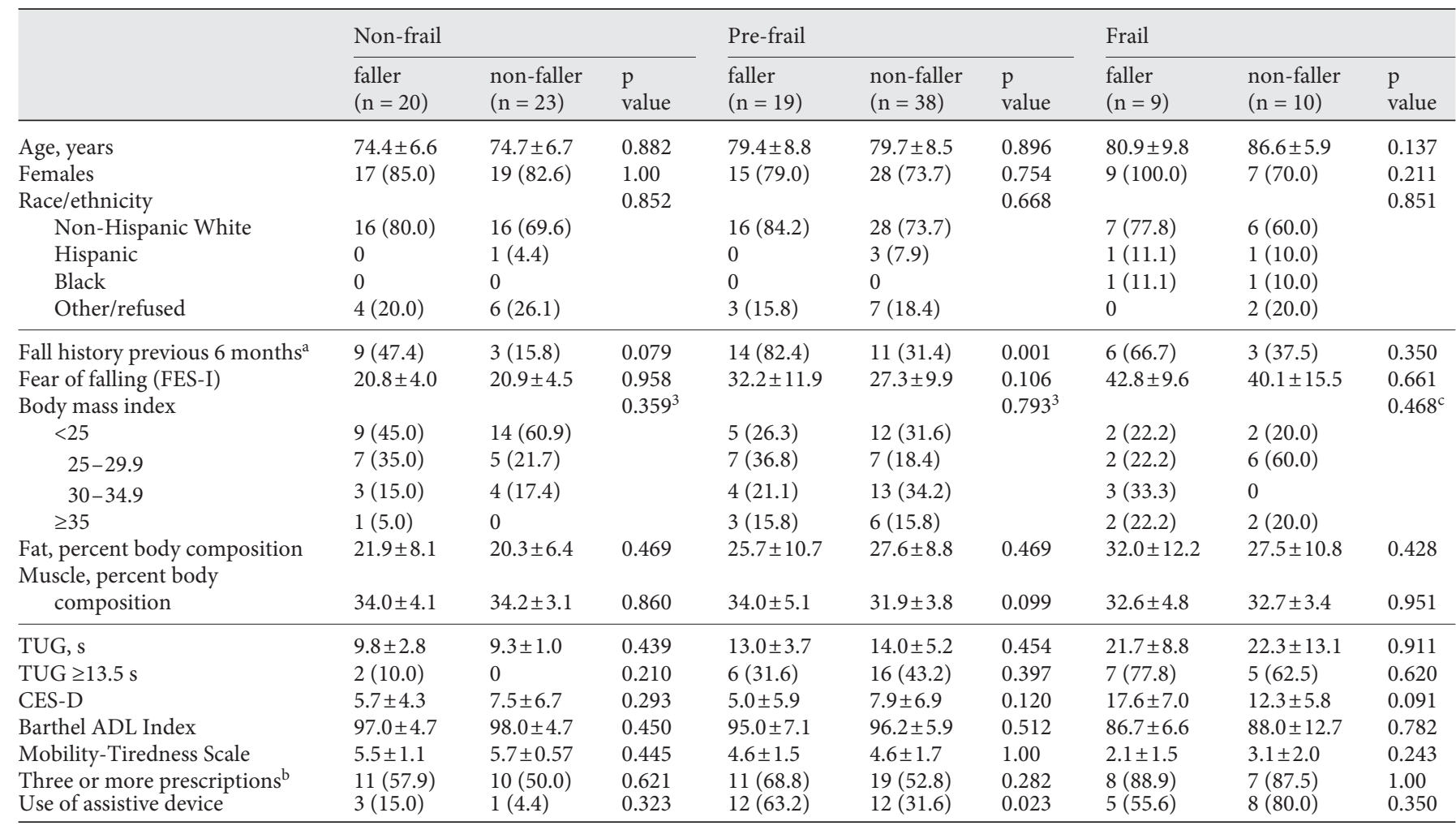

Data are presented as mean \pm SD or number (\%). ${ }^{a}$ Missing fall history in 5 non-frail, 5 pre-frail, 2 frail. ${ }^{b}$ Missing prescriptions in 4 non-frail, 4 pre-frail 2 frail. ${ }^{\mathrm{c}}$ Score test for trend.

Table 2. Balance parameters by incident faller status, stratified by frailty status

\begin{tabular}{|c|c|c|c|c|c|c|c|c|c|}
\hline & \multicolumn{3}{|l|}{ Non-frail } & \multicolumn{3}{|l|}{ Pre-frail } & \multicolumn{3}{|l|}{ Frail } \\
\hline & $\begin{array}{l}\text { faller } \\
(\mathrm{n}=20)\end{array}$ & $\begin{array}{l}\text { non-faller } \\
(\mathrm{n}=23)\end{array}$ & $\begin{array}{l}\text { effect size }^{\mathrm{a}} \\
\left(\mathrm{p} \text { value }^{\mathrm{b}}\right)\end{array}$ & $\begin{array}{l}\text { faller } \\
(n=19)\end{array}$ & $\begin{array}{l}\text { non-faller } \\
(\mathrm{n}=38)\end{array}$ & $\begin{array}{l}\text { effect size }^{\mathrm{a}} \\
\left(\mathrm{p} \text { value }^{\mathrm{b}} \text { ) }\right.\end{array}$ & $\begin{array}{l}\text { faller } \\
(\mathrm{n}=9)\end{array}$ & $\begin{array}{l}\text { non-faller } \\
(\mathrm{n}=10)\end{array}$ & $\begin{array}{l}\text { effect size }^{a} \\
\left(p \text { value }^{b}\right)\end{array}$ \\
\hline \multicolumn{10}{|l|}{ Eyes open } \\
\hline Ankle sway, $\operatorname{deg}^{2}$ & $3.5 \pm 3.0$ & $3.3 \pm 3.4$ & $0.065(0.232)$ & $7.2 \pm 5.3$ & $3.6 \pm 3.1$ & $0.898(0.007)$ & $8.0 \pm 10.8$ & $3.7 \pm 2.3$ & $0.563(0.564)$ \\
\hline Hip sway, deg $^{2}$ & $4.8 \pm 3.4$ & $3.9 \pm 4.3$ & $0.203(0.092)$ & $8.6 \pm 6.8$ & $4.1 \pm 3.0$ & $0.975(0.021)$ & $9.4 \pm 14.1$ & $4.6 \pm 4.0$ & $0.479(0.923)$ \\
\hline Hip sway-ankle sway ratio & $1.5 \pm 0.65$ & $1.9 \pm 0.86$ & $-0.134(0.057)$ & $1.3 \pm 1.00$ & $1.4 \pm 0.72$ & $-0.067(0.506)$ & $1.1 \pm 0.86$ & $1.3 \pm 0.75$ & $0.142(0.564)$ \\
\hline $\mathrm{COM}, \mathrm{cm}^{2}$ & $0.77 \pm 0.66$ & $0.69 \pm 0.65$ & $0.130(0.168)$ & $1.6 \pm 1.2$ & $0.66 \pm 0.38$ & $1.27(0.010)$ & $1.2 \pm 0.89$ & $0.68 \pm 0.44$ & $0.725(0.386)$ \\
\hline \multicolumn{10}{|l|}{$\mathrm{COM}, \mathrm{cm}$} \\
\hline Medial-lateral & $0.62 \pm 0.32$ & $0.56 \pm 0.32$ & $0.203(0.500)$ & $0.86 \pm 0.44$ & $0.59 \pm 0.33$ & $0.724(0.021)$ & $0.70 \pm 0.38$ & $0.53 \pm 0.30$ & $0.505(0.248)$ \\
\hline Anterior-posterior & $1.2 \pm 0.54$ & $1.1 \pm 0.68$ & $0.177(0.214)$ & $1.6 \pm 0.73$ & $1.2 \pm 0.44$ & $0.838(0.058)$ & $1.6 \pm 0.79$ & $1.5 \pm 0.58$ & $0.161(0.736)$ \\
\hline \multicolumn{10}{|l|}{ Eyes closed } \\
\hline Ankle sway, deg ${ }^{2}$ & $7.1 \pm 5.6$ & $5.2 \pm 3.6$ & $0.392(0.307)$ & $12.9 \pm 14.2$ & $8.1 \pm 5.9$ & $0.519(0.566)$ & $13.4 \pm 19.0$ & $14.9 \pm 20.1$ & $-0.076(0.923)$ \\
\hline Hip sway, $\operatorname{deg}^{2}$ & $6.6 \pm 2.9$ & $5.5 \pm 3.8$ & $0.312(0.223)$ & $16.8 \pm 20.6$ & $10.1 \pm 9.0$ & $0.485(0.409)$ & $16.7 \pm 23.1$ & $8.7 \pm 2.5$ & $0.455(0.630)$ \\
\hline Hip sway-ankle sway ratio & $1.4 \pm 0.94$ & $1.3 \pm 0.72$ & $0.134(0.922)$ & $1.5 \pm 0.86$ & $1.4 \pm 0.92$ & $0.105(0.591)$ & $1.5 \pm 0.72$ & $1.1 \pm 0.82$ & $0.446(0.083)$ \\
\hline $\mathrm{COM}, \mathrm{cm}^{2}$ & $1.4 \pm 0.96$ & $1.0 \pm 0.15$ & $0.438(0.268)$ & $3.2 \pm 3.4$ & $1.7 \pm 1.2$ & $0.694(0.247)$ & $2.5 \pm 2.3$ & $2.2 \pm 2.5$ & $0.131(0.847)$ \\
\hline \multicolumn{10}{|l|}{$\mathrm{COM}, \mathrm{cm}$} \\
\hline Medial-lateral & $0.82 \pm 0.36$ & $0.70 \pm 0.37$ & $0.337(0.233)$ & $1.2 \pm 0.92$ & $0.89 \pm 0.49$ & $0.390(0.435)$ & $1.1 \pm 0.61$ & $0.83 \pm 0.40$ & $0.444(0.564)$ \\
\hline Anterior-posterior & $1.6 \pm 0.63$ & $1.4 \pm 0.75$ & $0.307(0.214)$ & $2.3 \pm 1.4$ & $1.8 \pm 0.80$ & $0.480(0.294)$ & $2.0 \pm 0.85$ & $2.1 \pm 1.4$ & $-0.195(0.847)$ \\
\hline
\end{tabular}


Table 3. Gait parameters by incident faller status, stratified by frailty status

\begin{tabular}{|c|c|c|c|c|c|c|c|c|c|}
\hline & \multicolumn{3}{|l|}{ Non-frail } & \multicolumn{3}{|l|}{ Pre-frail } & \multicolumn{3}{|l|}{ Frail } \\
\hline & $\begin{array}{l}\text { faller } \\
(n=20)\end{array}$ & $\begin{array}{l}\text { non-faller } \\
(n=23)\end{array}$ & $\begin{array}{l}\text { effect size }{ }^{a} \\
\left(p \text { value }^{b}\right)\end{array}$ & $\begin{array}{l}\text { faller } \\
(\mathrm{n}=19)\end{array}$ & $\begin{array}{l}\text { non-faller } \\
(\mathrm{n}=38)\end{array}$ & $\begin{array}{l}\text { effect size }{ }^{a} \\
\left(p \text { value }^{b}\right)\end{array}$ & $\begin{array}{l}\text { faller } \\
(n=9)\end{array}$ & $\begin{array}{l}\text { non-faller } \\
(\mathrm{n}=10)\end{array}$ & $\begin{array}{l}\text { effect size }{ }^{a} \\
\left(p \text { value }^{b}\right)\end{array}$ \\
\hline \multicolumn{10}{|l|}{ Steady state } \\
\hline Speed, m/s & $1.18 \pm 0.18$ & $1.17 \pm 0.14$ & $0.079(0.592)$ & $0.93 \pm 0.26$ & $0.98 \pm 0.19$ & $-0.254(0.527)$ & $0.68 \pm 0.27$ & $0.75 \pm 0.47$ & $-0.195(0.930)$ \\
\hline Stride time, $s$ & $1.09 \pm 0.09$ & $1.08 \pm 0.10$ & $0.109(0.567)$ & $1.23 \pm 0.22$ & $1.17 \pm 0.16$ & $0.366(0.254)$ & $1.30 \pm 0.19$ & $1.29 \pm 0.22$ & $0.049(0.810)$ \\
\hline Stride length, m & $1.26 \pm 0.14$ & $1.24 \pm 0.09$ & $0.194(0.119)$ & $1.09 \pm 0.20$ & $1.12 \pm 0.15$ & $-0.154(0.640)$ & $0.84 \pm 0.25$ & $0.88 \pm 0.36$ & $-0.121(0.895)$ \\
\hline Double support, \% & $22.9 \pm 4.4$ & $21.4 \pm 3.9$ & $0.370(0.330)$ & $28.4 \pm 5.6$ & $25.9 \pm 5.8$ & $0.450(0.088)$ & $32.5 \pm 6.4$ & $30.5 \pm 9.8$ & $0.245(0.402)$ \\
\hline CV of speed, $\%$ & $4.2 \pm 2.8$ & $4.2 \pm 3.2$ & $-0.007(0.968)$ & $5.4 \pm 4.6$ & $5.0 \pm 3.6$ & $0.113(0.992)$ & $6.6 \pm 5.4$ & $5.0 \pm 2.3$ & $0.374(0.491)$ \\
\hline \multicolumn{10}{|l|}{ Initiation } \\
\hline Speed, m/s & $1.18 \pm 0.18$ & $1.18 \pm 0.14$ & $0.046(0.634)$ & $0.92 \pm 0.26$ & $0.98 \pm 0.20$ & $-0.234(0.556)$ & $0.67 \pm 0.27$ & $0.76 \pm 0.49$ & $-0.232(0.923)$ \\
\hline Stride time, s & $1.10 \pm 0.09$ & $1.09 \pm 0.11$ & $0.074(0.480)$ & $1.25 \pm 0.22$ & $1.18 \pm 0.15$ & $0.394(0.332)$ & $1.31 \pm 0.20$ & $1.32 \pm 0.24$ & $-0.008(0.772)$ \\
\hline Stride length, m & $1.26 \pm 0.15$ & $1.25 \pm 0.08$ & $0.134(0.150)$ & $1.09 \pm 0.20$ & $1.12 \pm 0.16$ & $-0.145(0.651)$ & $0.84 \pm .25$ & $0.90 \pm 0.40$ & $-0.178(0.847)$ \\
\hline Double support, \% & $23.2 \pm 4.3$ & $21.8 \pm 3.6$ & $0.348(0.301)$ & $30.0 \pm 5.2$ & $26.6 \pm 5.9$ & 0.604 (0.019) & $33.4 \pm 6.7$ & $32.8 \pm 12.6$ & $0.055(0.773)$ \\
\hline CV of speed, \% & $4.1 \pm 2.8$ & $3.4 \pm 1.5$ & $0.328(0.751)$ & $5.5 \pm 4.5$ & $5.4 \pm 4.0$ & $0.018(0.967)$ & $6.0 \pm 2.7$ & $7.5 \pm 7.2$ & $-0.289(0.791)$ \\
\hline
\end{tabular}

Data are presented as mean $\pm \mathrm{SD}$ or as stated. $\mathrm{CV}=$ Coefficient of variation $=$ mean/SD. ${ }^{\mathrm{a}}$ Cohen's d. ${ }^{\mathrm{b}} \mathrm{Mann}-\mathrm{Whitney} \mathrm{U}$ test.

open trials, we observed significantly larger mean values in fallers for ankle sway $(\mathrm{d}=0.898, \mathrm{p}=0.007)$, hip sway $(\mathrm{d}=0.975, \mathrm{p}=0.02)$, and COM sway $(\mathrm{d}=1.27, \mathrm{p}=0.01)$. The same measures in the frail group produced relatively large effect sizes, which were not statistically significant (ankle sway $\mathrm{d}=0.563$, hip sway $\mathrm{d}=0.479$, COM sway $\mathrm{d}=$ $0.725)$. Analogous measures in eyes closed trials were not significantly different by faller status.

\section{Gait}

Table 3 shows wearable sensor-based gait parameters, comparing fallers with non-fallers separately for each frailty category. In the pre-frail group, fallers had a significantly greater mean double support during gait initiation $(\mathrm{d}=0.604, \mathrm{p}=0.02)$; this difference was not statistically significant for double support percent during steadystate gait $(\mathrm{d}=0.450, \mathrm{p}=0.09)$. No other significant associations between gait parameters and faller status were observed, including several not shown, such as gait cycle time, shin speed, knee range of motion, and trunk sway during walking.

\section{Physical Activity}

Table 4 shows wearable sensor-based PA parameters, comparing fallers with non-fallers separately for each frailty category. In the frail group, we observed relatively large effect sizes for walking parameters, indicating larger mean values in fallers: walking time percent $(\mathrm{d}=1.08$, $\mathrm{p}=0.07)$, mean walking bout duration $(\mathrm{d}=1.16, \mathrm{p}=0.03)$, 90th percentile of walking bout duration $(\mathrm{d}=0.960, \mathrm{p}=$ $0.04)$, walking bout duration variability $(\mathrm{d}=1.10, \mathrm{p}=$
$0.05)$, and steps per $24 \mathrm{~h}(\mathrm{~d}=0.991, \mathrm{p}=0.09)$. Although few standing and sitting parameter differences by faller status were significant, the pattern of effect sizes suggests that non-fallers in both the prefrail and frail groups have longer episodes for both standing and sitting and greater episode duration variability for standing, compared to fallers. For example, mean standing bout duration in prefrail $(\mathrm{d}=-0.452)$ and frail $(\mathrm{d}=-0.394)$, as well as mean sitting bout duration in pre-frail $(\mathrm{d}=-0.392)$ and frail $(d=-0.977)$, all showed relatively large negative effect sizes.

\section{Adjusted Model}

In the non-frail sample, the only independent, significant predictor of faller status observed was history of a fall in the previous 6 months $(\mathrm{OR}=4.8,95 \%$ CI $1.02-22.6$, $\mathrm{p}=0.047$ ). Table 5 shows the results of sequential multiple logistic regression modeling in the subsample of frail and pre-frail subjects combined. In the frail/pre-frail sample, most variables that were candidates based on the results in tables $2-4$, had $\mathrm{p}<0.20$ in univariate logistic regression and were included in subsequent blocks of variables for multiple regression. Excluded due to collinearity were steps per $24 \mathrm{~h}$ (collinear with walking time percent), double support during gait initiation (collinear with double support during steady state), and the 90th percentile bout duration for walking, standing, or sitting (collinear with respective mean duration).

In model 1 of the balance parameter block, only COM sway was an independent predictor $(\mathrm{OR}=4.5,95 \% \mathrm{CI}$ $1.7-12.0, \mathrm{p}=0.003$ ). In model 2 , the addition of the only candidate gait parameter, double support percent, yield- 
Table 4. Physical activity parameters $(48 \mathrm{~h})$ by incident faller status, stratified by frailty status

\begin{tabular}{|c|c|c|c|c|c|c|c|c|c|}
\hline & \multicolumn{3}{|l|}{ Non-frail } & \multicolumn{3}{|l|}{ Pre-frail } & \multicolumn{3}{|l|}{ Frail } \\
\hline & $\begin{array}{l}\text { faller } \\
(\mathrm{n}=20)\end{array}$ & $\begin{array}{l}\text { non-faller } \\
(\mathrm{n}=23)\end{array}$ & $\begin{array}{l}\text { effect size } \\
\left.\text { (p value }^{\mathrm{b}}\right)\end{array}$ & $\begin{array}{l}\text { faller } \\
(\mathrm{n}=19)\end{array}$ & $\begin{array}{l}\text { non-faller } \\
(\mathrm{n}=38)\end{array}$ & 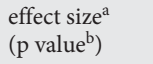 & $\begin{array}{l}\text { faller } \\
(\mathrm{n}=9)\end{array}$ & $\begin{array}{l}\text { non-faller } \\
(\mathrm{n}=10)\end{array}$ & $\begin{array}{l}\text { effect size } \\
\left(p \text { value }^{\mathrm{b}}\right)\end{array}$ \\
\hline \multicolumn{10}{|l|}{ Walking } \\
\hline Walking during $48 \mathrm{~h}, \%$ & $6.9 \pm 3.2$ & $7.7 \pm 3.2$ & $-0.254(0.543)$ & $5.4 \pm 1.8$ & $5.3 \pm 2.6$ & $0.035(0.518)$ & $5.2 \pm 3.1$ & $2.4 \pm 2.0$ & $1.08(0.072)$ \\
\hline Mean walking bout duration, $s$ & $39.3 \pm 16.1$ & $42.9 \pm 16.8$ & $-0.218(0.551)$ & $39.6 \pm 15.9$ & $39.0 \pm 12.3$ & $0.040(0.530)$ & $45.4 \pm 12.4$ & $30.2 \pm 13.7$ & $1.16(0.034)$ \\
\hline Walking bout duration, 90th percentile, $s$ & $70.6 \pm 28.9$ & $78.0 \pm 27.3$ & $-0.263(0.368)$ & $84.0 \pm 36.4$ & $78.8 \pm 28.5$ & $0.164(0.804)$ & $97.0 \pm 39.5$ & $61.8 \pm 34.1$ & $0.960(0.041)$ \\
\hline Walking bout duration variability, s & $89.1 \pm 76.3$ & $87.9 \pm 80.4$ & $0.015(0.715)$ & $56.3 \pm 37.6$ & $59.6 \pm 44.1$ & $-0.078(0.684)$ & $49.2 \pm 20.2$ & $28.4 \pm 17.6$ & $1.10(0.050)$ \\
\hline Steps per $24 \mathrm{~h}, \mathrm{n}$ & $5,969 \pm 3,730$ & $6,147 \pm 2,671$ & $-0.056(0.644)$ & $3,790 \pm 1,589$ & $3,981 \pm 2,488$ & $-0.086(0.697)$ & $3,644 \pm 2,568$ & $1,625 \pm 1,407$ & $0.991(0.086)$ \\
\hline \multicolumn{10}{|l|}{ Standing } \\
\hline Standing during $48 \mathrm{~h}, \%$ & $17.3 \pm 4.8$ & $17.1 \pm 4.9$ & $0.167(0.798)$ & $14.2 \pm 5.2$ & $13.9 \pm 4.2$ & $0.076(0.763)$ & $13.6 \pm 4.3$ & $10.8 \pm 5.2$ & $0.590(0.165)$ \\
\hline Mean standing bout duration, $\mathrm{s}$ & $65.2 \pm 26.7$ & $59.9 \pm 17.6$ & $0.235(0.361)$ & $56.0 \pm 12.9$ & $63.6 \pm 18.7$ & $-0.452(0.124)$ & $66.2 \pm 10.4$ & $73.0 \pm 21.3$ & $-0.394(0.514)$ \\
\hline Standing bout duration, 90 th percentile, $s$ & $155.8 \pm 68.5$ & $143.2 \pm 38.5$ & $0.230(0.450)$ & $129.5 \pm 26.7$ & $147.6 \pm 38.2$ & $-0.522(0.055)$ & $157.0 \pm 32.0$ & $164.9 \pm 51.7$ & $-0.180(0.744)$ \\
\hline Standing bout duration variability, s & $99.0 \pm 43.6$ & $94.6 \pm 47.1$ & $0.098(0.635)$ & $82.6 \pm 34.8$ & $100.8 \pm 58.3$ & $-0.353(0.154)$ & $89.2 \pm 13.2$ & $110.4 \pm 67.5$ & $-0.424(0.744)$ \\
\hline \multicolumn{10}{|l|}{ Sitting } \\
\hline Sitting during $48 \mathrm{~h}, \%$ & $35.1 \pm 7.7$ & $33.9 \pm 10.1$ & $0.133(0.961)$ & $41.5 \pm 11.5$ & $38.6 \pm 10.3$ & $0.269(0.371)$ & $41.1 \pm 6.5$ & $45.2 \pm 16.6$ & $-0.319(0.568)$ \\
\hline Mean sitting bout duration, $\mathrm{s}$ & $210.9 \pm 81.4$ & $224.6 \pm 108.4$ & $-0.142(0.697)$ & $254.0 \pm 93.3$ & $310.1 \pm 162.7$ & $-0.392(0.344)$ & $298.8 \pm 57.6$ & $429.4 \pm 175.4$ & $-0.977(0.041)$ \\
\hline Sitting bout duration, 90 th percentile, $s$ & $545.1 \pm 224.0$ & $625.7 \pm 350.5$ & $-0.270(0.511)$ & $613.8 \pm 312.9$ & $876.0 \pm 546.1$ & $-0.546(0.046)$ & $776 \pm 195$ & $1,148 \pm 601$ & $-0.815(0.121)$ \\
\hline Sitting bout duration variability, s & $512.6 \pm 214.9$ & $473.4 \pm 175.0$ & $0.202(0.422)$ & $628.2 \pm 216.8$ & $629.8 \pm 280.1$ & $-0.006(0.804)$ & $594 \pm 141$ & $910 \pm 494$ & $-0.849(0.050)$ \\
\hline \multicolumn{10}{|l|}{ Lying } \\
\hline Lying during $48 \mathrm{~h}$, \% & $40.1 \pm 8.1$ & $41.3 \pm 9.2$ & $-0.136(0.836)$ & $38.9 \pm 12.5$ & $42.2 \pm 9.7$ & $-0.309(0.184)$ & $40.1 \pm 9.2$ & $41.6 \pm 18.3$ & $-0.100(0.624)$ \\
\hline Mean lying bout duration, $s$ & $1,930 \pm 860$ & $1,941 \pm 864$ & $-0.012(0.865)$ & $2,778 \pm 1,366$ & $2,734 \pm 1,587$ & $0.029(0.490)$ & $2,289 \pm 598$ & $3,239 \pm 1,873$ & $-0.668(0.414)$ \\
\hline Lying bout duration, $90^{\text {th }}$ percentile, $\mathrm{s}$ & $5,392 \pm 2,979$ & $5,993 \pm 2,926$ & $-0.203(0.450)$ & $8,438 \pm 5,221$ & $7,800 \pm 5,549$ & $0.117(0.468)$ & $6,718 \pm 1,777$ & $8,665 \pm 4,463$ & $-0.561(0.462)$ \\
\hline Lying bout duration variability, s & $3,572 \pm 1,483$ & $3,445 \pm 1,652$ & $0.081(0.679)$ & $3,856 \pm 1,828$ & $4,289 \pm 3,200$ & $-0.154(0.697)$ & $2,982 \pm 958$ & $3,813 \pm 1,780$ & $-0.572(0.289)$ \\
\hline Postural transitions per $24 \mathrm{~h}, \mathrm{n}$ & $163.7 \pm 50.1$ & $152.2 \pm 47.8$ & $0.236(0.450)$ & $158.2 \pm 57.1$ & $126.8 \pm 41.1$ & $0.668(0.063)$ & $124.5 \pm 23.4$ & $106.2 \pm 44.8$ & $0.505(0.191)$ \\
\hline
\end{tabular}

Data are presented as mean \pm SD or as stated. ${ }^{a}$ Cohen's d. ${ }^{b}$ Mann-Whitney U test.

Table 5. Multiple logistic regression models predicting having any incident fall in frail and pre-frail subjects combined

\begin{tabular}{|c|c|c|c|c|c|c|}
\hline Variable & Univariate & $\begin{array}{l}\text { Model } 1 \\
\text { balance block } \\
(\mathrm{n}=67)\end{array}$ & $\begin{array}{l}\text { Model } 2 \\
\text { gait block } \\
(\mathrm{n}=62)\end{array}$ & $\begin{array}{l}\text { Model } 3 \\
\text { PA block } \\
(\mathrm{n}=65)\end{array}$ & $\begin{array}{l}\text { Model } 4 \\
\text { other block } \\
(\mathrm{n}=60)\end{array}$ & $\begin{array}{l}\text { Model } 5 \\
\text { parsimonious } \\
(\mathrm{n}=60)\end{array}$ \\
\hline Ankle sway EO $\left(\mathrm{deg}^{2}\right)$ & $1.2(1.0-1.5)^{\mathrm{c}}$ & $1.0(0.85-1.2)$ & & & & \\
\hline Hip sway EO $\left(\mathrm{deg}^{2}\right)$ & $1.2(1.0-1.3)^{\mathrm{b}}$ & $0.99(0.86-1.1)$ & & & & \\
\hline Double support, steady state (\%) & $1.1(0.98-1.2)^{\mathrm{e}}$ & & $1.0(0.94-1.2)$ & & & \\
\hline Walking bout duration variability (s) & $1.0(0.99-1.0)$ & & & & & \\
\hline Mean standing bout duration (s) & $0.97(0.95-1.0)^{\mathrm{d}}$ & & & $0.94(0.90-0.99)^{\mathrm{c}}$ & $0.91(0.84-0.98)^{\mathrm{b}}$ & $0.94(0.91-0.99)^{\mathrm{b}}$ \\
\hline Mean sitting bout duration (s) & $0.99(0.99-1.0)^{\mathrm{c}}$ & & & $0.99(0.98-1.0)$ & & \\
\hline Sitting bout duration variability (s) & $0.99(0.99-1.0)$ & & & & & \\
\hline Postural transitions per $24 \mathrm{~h}(\mathrm{n})$ & $1.0(1.0-1.0)^{\mathrm{c}}$ & & & $1.0(0.99-1.0)$ & & \\
\hline Age (years) & $0.98(0.93-1.0)$ & & & & & \\
\hline Fall history previous 6 months $(\mathrm{Y} / \mathrm{N})$ & $6.9(2.2-21.2)^{\mathrm{a}}$ & & & & $6.7(1.3-34.7)^{\mathrm{c}}$ & $7.3(1.5-36.4)^{\mathrm{c}}$ \\
\hline Model pseudo $\mathrm{R}^{2}$ & & 0.186 & 0.189 & 0.327 & 0.478 & 0.427 \\
\hline Model AUC & & 0.705 & 0.738 & 0.842 & 0.907 & 0.882 \\
\hline Model AIC ${ }^{f}$ & & 68.7 & 66.6 & 63.1 & 54.4 & 54.8 \\
\hline
\end{tabular}

Values indicate OR $(95 \% \mathrm{CI}) . \mathrm{EO}=$ Eyes open. ${ }^{\mathrm{a}} \mathrm{p}<0.001 .{ }^{\mathrm{b}} \mathrm{p}<0.01 .{ }^{\mathrm{c}} \mathrm{p}<0.05 .{ }^{\mathrm{d}} \mathrm{p}<0.10 .{ }^{\mathrm{e}} \mathrm{p}<0.20 .{ }^{\mathrm{f}}$ From models on a subset of 55 subjects with no missing variables. 
ed similar estimates for COM sway $(\mathrm{OR}=3.8,95 \% \mathrm{CI}$ $1.9-7.8, \mathrm{p}<0.001)$. Model 3 introduced a block of candidate PA parameters; mean walking bout duration and mean standing bout duration were both significant predictors, indicating an increased risk of falling with increasing walking episode duration and a decreased risk of falling with increasing standing episode duration. In model 3, the association with COM sway was strengthened considerably, indicating possible confounding by PA. Model 4 shows that fall history in previous 6 months is a strong predictor of future falls, but its inclusion did not alter the estimates of other independent predictors substantially. The use of assistive devices had a large OR point estimate, but an extremely wide $\mathrm{CI}$, indicating uncertainty in this estimate $(\mathrm{p}=0.20)$.

Finally, model 5 demonstrates that exclusion of the non-significant covariates from model 4 , particularly use of assistive devices, made very little change to the estimates of other independent predictors. Furthermore, model 5 in frail and pre-frail separately produced estimated ORs similar to or stronger than (though not always statistically significant) model 5 in pre-frail combined for COM sway (OR 8.8, $\mathrm{p}<0.001$ pre-frail; OR 13.9, $\mathrm{p}=0.10$ frail), mean walking bout duration (OR $1.1, \mathrm{p}=0.02$ prefrail; OR 1.5, $\mathrm{p}=0.01$ frail), and mean standing bout duration (OR $0.95, \mathrm{p}=0.03$ pre-frail; OR $0.77, \mathrm{p}=0.07$ frail). In model 5 for frail only, an OR for fall history in previous 6 months could not be estimated because it predicted prospective falls perfectly.

For model 5 in pre-frail and frail combined, the standardized ORs for continuous parameters were $\mathrm{OR}_{\mathrm{S}}=4.6$ $\mathrm{COM}$ sway, $\mathrm{OR}_{\mathrm{S}}=3.4$ mean walking bout duration, $\mathrm{OR}_{\mathrm{S}}=0.4$ mean standing bout duration, and $\mathrm{OR}_{\mathrm{S}}=2.7$ for fall history in previous 6 months, indicating that the relative importance of the sensor-based parameters is COM sway $>$ walking bout duration $>$ fall history $>$ standing bout duration. Inspection of the pseudo $\mathrm{R}^{2}$ across models shows better prediction of the outcome with each addition of independent predictors, rising to 0.478 in model 4. The AUC increased markedly from 0.705 in model 1 to 0.907 in model 4 , indicating that model 4 is highly accurate in discriminating between fallers and nonfallers. A lower AIC indicates better quality in terms of goodness of fit or the presence of fewer covariates without loss of fit. The AIC decrease between model 1 and model 4 indicates increasing model quality in terms of goodness of fit without superfluous covariates that do not contribute to fit. For model 5, all three indicators are slightly less optimal, suggesting that the more parsimonious model does not improve predictive value or goodness of fit.

Falls and Wearable Sensor-Based Measures in Older Adults

\section{Discussion}

This study examined the most sensitive sensor-derived $\mathrm{PA}$, gait, and balance parameters for prediction of prospective falls during a 6-month follow-up period in community dwelling elders stratified based on frailty status. A strength of the study lies in the fact that the measures were conducted in the home and community settings, which allowed for inclusion of nearly homebound participants who are often excluded in clinic-based studies. The results suggest that performance-based tests such as gait trials and TUG are insensitive predictors of future falls in particular among frail and pre-frail older adults, whereas certain balance and PA parameters related to walking and standing may be useful fall risk predictors in populations with indicators of frailty.

With the exception of balance measures and history of falls, none of the demographic, questionnaires, or performance-based tests (i.e., gait trials, TUG test) discriminated between future fallers and non-fallers. When categorizing older adults based on frailty status, we did not observe an association between fall risk and gait velocity or gait measures other than double support percent, which was not a significant predictor in adjusted models. Previous studies have described increased fall risk associated with slower gait speed, swing, double-support percent, swing time variability, and stride length variability [33, 34]. However, these studies did not stratify or adjust for frailty status to the best of our knowledge, and often excluded frail, home-bound, older adults. Although there are reports of TUG as a significant predictor of fall risk [35], a recent systematic review concluded that performance-based tests such as TUG had poor to moderate accuracy for predicting future falls, especially in higherfunctioning older people [36].

Our results suggest that among frail and pre-frail older adults, balance and PA parameters are predictive of fall risk, but gait parameters are not. The findings indicate that COM sway, mean walking bout duration, and mean standing bout duration, are sensor-based measures that could enhance the accuracy of fall risk assessment in frail elders. We found that increased COM sway (each $\mathrm{cm}^{2}$ ) was associated with a roughly 4 - to 6 -fold increased odds of a fall over 6 months, increased mean walking bout duration (each second) with a $10 \%$ increased odds, and increased mean standing bout duration (each second) with a $6 \%$ decreased odds, adjusted for history of a fall in the previous 6 months. Of particular interest is the finding that increased PA (as measured by bout duration) was associated with a greater occurrence of falls, which we ob- 
served in the frail group (unadjusted and adjusted results) and the pre-frail group (adjusted results), consistent with the possibility that frail and pre-frail elders who do not avoid PA may experience more falls. None of the sensorderived parameters were sensitive to predict prospective falls in the non-frail group, which may indicate that falls in non-frail people are not dependent on motor performance and functional status.

We did not observe an increased risk of falling with increasing frailty category, which is inconsistent with numerous previous reports $[37,38]$. One explanation could be that the non-frail participants in our study were unusually active, had a lower fear of falling, took more risks or were less cautious, and experienced a higher rate of falls than typically observed in non-frail samples. For example, in the Study of Osteoporotic Fractures, only 8\% of non-frail women (mean age 75 , same as our non-frail sample) reported two or more falls over a year, compared to $30 \%$ in our study over 6 months [37]. Another contributing explanation could be that the frail participants exercised greater caution, which could have been heighted by a Hawthorne effect of participating in a study whose goals included surveillance of falls. Such caution may have a greater preventive effect in pre-frail participants, because many frail persons have reached a level of weakness and mobility deficit, where a high fall risk is difficult to mitigate. Finally, fall rates in the frail group of our study may have been reduced by the exclusion of those with a Mini-Mental State Examination score of 23 or lower, movement disorders such as Parkinson's disease or multiple sclerosis, or a recent stroke. These exclusions were made to assure that subjects could complete the body-worn sensor assessments, but also removed more complicated and higher-risk subjects.

\section{Limitations and Future Research}

There are several limitations to consider in the interpretation of these findings. First, our sample size included 57 pre-frail and 19 frail participants. The power to detect differences between fallers and non-fallers in participant characteristics and sensor-based measures may have been limited in the frail group; as a result, we combined pre-frail and frail for multiple logistic regression predicting a prospective faller. Although this combination was supported by a pattern of effect sizes for faller status that were comparable or in the same direction between pre-frail and frail, there were more exceptions to this pattern among PA parameters than gait or balance parameters. However, the final adjusted models in frail and pre-frail separately were comparable to and corroborated the model with frail and pre-frail combined. Second, our sample was predominantly women; although we did not observe a difference in fall risk by sex, our model parameter estimates may have a limited generalizability to a population with a more balanced sex composition. Third, the study exclusions for cognitive impairment, movement disorders, and stroke may limit generalizability of our findings to populations without these comorbidities. Fourth, we recruited participants using a convenience sample technique designed to oversample frail and pre-frail community-dwelling elders. Therefore, the sample may not adequately represent the general population of community-dwelling older adults, and the effects we estimated should be validated in a larger probability sample. Fifth, we used the most widely adopted Fried frailty criteria, which has been associated with numerous poor outcomes. However, there is to date no clear consensus regarding the definition of frailty [39]. It is possible that a broader frailty concept including cognitive, psychological, or social components (such as the LASA frailty instrument [38]) or a continuous measure (such as the Rockwood Index [40]), could have altered the moderating effect we observed for frailty. A sixth potential limitation is the 48-hour PA assessment period, which may not cover day-to-day variability. Given that PA in older adults is less variable and high day-to-day reliability of PA assessment has been reported for older adults ( $>60$ years) [41], we believe that 48-hour monitoring in our study was most likely sufficient to document habitual PA. Seventh, we reported gait parameters that have been most commonly reported and associated with falls in previous studies [33, 42, 43]. However, there are some other gait parameters (e.g., fractal gait analysis or local dynamic stability) that require nonlinear analysis of a large sample of gait data. Due to the limitations of our experimental setup, we were not able to perform a long-distance walking test, and our data were not sufficiently large for nonlinear gait analysis. Additional frequency-based gait (quality) parameters can be extracted from free-living activity data using nonlinear frequency analysis, which we plan to assess in future analyses. Finally, about $5 \%$ of subjects who recreated their fall diaries at the follow-up visit may have been prone to recall bias. We would expect that in our cohort of cognitively intact subjects, all serious falls requiring medical intervention would have been reported, whereas minor falls may have been underreported in the small number of participants requiring assistance to fill out diaries.

We included assistive device (canes and walkers) users who used their regular devices during gait trials, which may have minimized the detection of gait deficits [44]. Gait in older adults who use a walking assistive device is 
more irregular and unstable than gait in independently mobile older adults. Assistive device users have a better gait when using their device than when walking without it. Gait performance significantly improves when assessed with walking aids, both for canes (increased stride time and length, decreased cadence, and stride length variability) and walkers (increased gait speed and stride length, decreased base of support, and double support) [45]. The need for assistive devices is an indicator of conditions that increase fall risk, as replicated in our results, but their use generally mitigates fall risk. We believe that gait assessment with an assistive device most closely characterizes the nature of participants' gait during the time of fall ascertainment.

\section{Conclusions}

Importantly, we found that the association between motor performance and risk of falling is dependent on frailty status. While prospective falls in non-frail older adults are not dependent on motor performance, balance deficits and inability of ample walking and ample standing are strong predictors of prospective falls in pre-frail and frail older adults. Surprisingly, while gait is deteriorated by increasing frailty status, it is not a predictor of falls when older adults are categorized based on frailty status. Our findings highlight the potential value of wearable sensor technology as a practical tool for assessing fall risk in the home setting even among frail older adults with limited mobility. Using these variables in a future index and validating this index in a larger cohort could provide a useful fall prediction tool especially for pre-frail and frail older adults.

\section{Acknowledgments}

The project described was supported in part by an award (2R42AG032748) from the National Institute on Aging, and the Arizona Center on Aging. The content is solely the responsibility of the authors and does not necessarily represent the official views of the National Institute on Aging or the National Institutes of Health. We thank the Arizona Frailty Cohort coordinator Marilyn Gilbert and student workers for the recruitment of participants and collection of data, as well as study participants for their kind participation. The clinical trial was registered with ClinicalTrials.gov, identifier NCT01880229. The sponsor had no role in the design or conduct of this study; collection, management, analysis, or interpretation of the data; or preparation, review, or approval of the manuscript.

\section{References}

$>_{1}$ Tinetti ME, Williams CS: Falls, injuries due to falls, and the risk of admission to a nursing home. N Engl J Med 1997;337:1279-1284.

2 Guideline for the prevention of falls in older persons. American Geriatrics Society, British Geriatrics Society, and American Academy of Orthopaedic Surgeons Panel on Falls Prevention. J Am Geriatr Soc 2001;49:664-672.

3 Rubenstein LZ: Falls in older people: epidemiology, risk factors and strategies for prevention. Age Ageing 2006;35(suppl 2):ii37ii41.

4 Horan MA, Clague JE: Injury in the aging: recovery and rehabilitation. Br Med Bull 1999; 55:895-909.

5 Bloch F, Blandin M, Ranerison R, Claessens YE, Rigaud AS, Kemoun G: Anxiety after a fall in elderly subjects and subsequent risk of developing post traumatic stress disorder at two months. A pilot study. J Nutr Health Aging 2014;18:303-306.

6 Najafi B, Armstrong DG, Mohler J: Novel wearable technology for assessing spontaneous daily physical activity and risk of falling in older adults with diabetes. J Diabetes Sci Technol 2013;7:1147-1160.
7 Lord SR, Menz HB, Sherrington C: Home environment risk factors for falls in older people and the efficacy of home modifications. Age Ageing 2006;35(suppl 2):ii55-ii59.

$\checkmark 8$ Weiss A, Brozgol M, Dorfman M, Herman T, Shema S, Giladi N, Hausdorff JM: Does the evaluation of gait quality during daily life provide insight into fall risk? A novel approach using 3-day accelerometer recordings. Neurorehabil Neural Repair 2013;27:742-752.

-9 Shany T, Redmond SJ, Marschollek M, Lovell $\mathrm{NH}$ : Assessing fall risk using wearable sensors: a practical discussion. A review of the practicalities and challenges associated with the use of wearable sensors for quantification of fall risk in older people. Z Gerontol Geriatr 2012;45:694-706.

10 Howcroft J, Kofman J, Lemaire ED: Review of fall risk assessment in geriatric populations using inertial sensors. J Neuroeng Rehabil 2013;10:91.

11 Rispens SM, van Schooten KS, Pijnappels M, Daffertshofer A, Beek PJ, van Dieen JH: Do extreme values of daily-life gait characteristics provide more information about fall risk than median values? JMIR Res Protoc 2015;4:e4.
12 Weiss A, Herman T, Giladi N, Hausdorff JM: Objective assessment of fall risk in Parkinson's disease using a body-fixed sensor worn for 3 days. PLoS One 2014;9:e96675.

13 Ihlen EA, Weiss A, Helbostad JL, Hausdorff JM: The discriminant value of phase-dependent local dynamic stability of daily life walking in older adult community-dwelling fallers and nonfallers. Biomed Res Int 2015;2015: 402596.

14 Klenk J, Kerse N, Rapp K, Nikolaus T, Becker C, Rothenbacher D, Peter R, Denkinger MD: Physical activity and different concepts of fall risk estimation in older people - results of the ActiFE-Ulm Study. PLoS One 2015;10: e0129098.

15 Fried LP, Tangen CM, Walston J, Newman AB, Hirsch C, Gottdiener J, Seeman T, Tracy R, Kop WJ, Burke G: Frailty in older adults evidence for a phenotype. J Gerontol A Biol Sci Med Sci 2001;56:M146-M157.

16 Collard RM, Boter H, Schoevers RA, Oude Voshaar RC: Prevalence of frailty in community-dwelling older persons: a systematic review. J Am Geriatr Soc 2012;60:1487-1492.
Falls and Wearable Sensor-Based Measures in Older Adults 
17 Schwenk M, Mohler J, Wendel C, D’Huyvetter K, Fain M, Taylor-Piliae R, Najafi B: Wearable sensor-based in-home assessment of gait, balance, and physical activity for discrimination of frailty status: baseline results of the Arizona frailty cohort study. Gerontology 2015;61: 258-267.

18 Folstein MF, Folstein SE, McHugh PR: 'Minimental state'. A practical method for grading the cognitive state of patients for the clinician. J Psychiatr Res 1975;12:189-198.

19 Fieo RA, Mortensen EL, Rantanen T, Avlund $\mathrm{K}$ : Improving a measure of mobility-related fatigue (the Mobility-Tiredness Scale) by establishing item intensity. J Am Geriatr Soc 2013;61:429-433.

20 Orme JG, Reis J, Herz EJ: Factorial and discriminant validity of the Center for Epidemiological Studies Depression (CES-D) Scale. J Clin Psychol 1986;42:28-33.

-21 Mahoney FI, Barthel DW: Functional evaluation: the Barthel Index. Md State Med J 1965; 14:61-65.

-22 Yardley L, Beyer N, Hauer K, Kempen G, Piot-Ziegler C, Todd C: Development and initial validation of the Falls Efficacy ScaleInternational (FES-I). Age Ageing 2005;34: 614-619.

23 Podsiadlo D, Richardson S: The timed 'Up \& Go': a test of basic functional mobility for frail elderly persons. J Am Geriatr Soc 1991;39: 142-148.

-24 Bandeen-Roche K, Xue QL, Ferrucci L, Walston J, Guralnik JM, Chaves P, Zeger SL, Fried LP: Phenotype of frailty: characterization in the women's health and aging studies. J Gerontol A Biol Sci Med Sci 2006;61:262266.

-25 Taylor HL, Jacobs DR Jr, Schucker B, Knudsen J, Leon AS, Debacker G: A questionnaire for the assessment of leisure time physical activities. J Chronic Dis 1978;31:741-755.

-26 Lamb SE, Jorstad-Stein EC, Hauer K, Becker C: Development of a common outcome data set for fall injury prevention trials: the Prevention of Falls Network Europe consensus. J Am Geriatr Soc 2005;53:1618-1622.
7 Campbell AJ, Robertson MC, Gardner MM, Norton RN, Tilyard MW, Buchner DM: Randomised controlled trial of a general practice programme of home based exercise to prevent falls in elderly women. BMJ 1997;315: 1065-1069.

28 Najafi B, Horn D, Marclay S, Crews RT, Wu S, Wrobel JS: Assessing postural control and postural control strategy in diabetes patients using innovative and wearable technology. J Diabetes Sci Technol 2010;4:780-791.

29 Najafi B, Helbostad JL, Moe-Nilssen R, Zijlstra W, Aminian K: Does walking strategy in older people change as a function of walking distance? Gait Posture 2009;29:261-266.

-30 Najafi B, Lee-Eng J, Wrobel JS, Goebel R: Estimation of center of mass trajectory using wearable sensors during golf swing. J Sports Sci Med 2015;14:354-363.

-31 Toosizadeh N, Lei H, Schwenk M, Sherman SJ, Sternberg E, Mohler J, Najafi B: Does integrative medicine enhance balance in aging adults? Proof of concept for the benefit of electroacupuncture therapy in Parkinson's disease. Gerontology 2015;61:3-14.

32 Toosizadeh N, Mohler J, Lei H, Parvaneh S, Sherman S, Najafi B: Motor performance assessment in Parkinson's disease: association between objective in-clinic, objective inhome, and subjective/semi-objective measures. PLoS One 2015;10:e124763.

-33 Verghese J, Holtzer R, Lipton RB, Wang C: Quantitative gait markers and incident fall risk in older adults. J Gerontol A Biol Sci Med Sci 2009;64:896-901.

-34 Beauchet O, Annweiler C, Allali G, Berrut G, Herrmann FR, Dubost V: Recurrent falls and dual task-related decrease in walking speed: is there a relationship? J Am Geriatr Soc 2008; 56:1265-1269.

35 Greene BR, Doheny EP, Walsh C, Cunningham C, Crosby L, Kenny RA: Evaluation of falls risk in community-dwelling older adults using body-worn sensors. Gerontology 2012; 58:472-480.
6 Schoene D, Wu SM, Mikolaizak AS, Menant JC, Smith ST, Delbaere K, Lord SR: Discriminative ability and predictive validity of the timed up and go test in identifying older people who fall: systematic review and meta-analysis. J Am Geriatr Soc 2013;61:202-208.

37 Ensrud KE, Ewing SK, Taylor BC, Fink HA, Stone KL, Cauley JA, Tracy JK, Hochberg MC, Rodondi N, Cawthon PM: Frailty and risk of falls, fracture, and mortality in older women: the study of osteoporotic fractures. J Gerontol A Biol Sci Med Sci 2007;62:744-751.

38 de Vries OJ, Peeters GM, Lips P, Deeg DJ: Does frailty predict increased risk of falls and fractures? A prospective population-based study. Osteoporos Int 2013;24:2397-2403.

39 Rodriguez-Manas L, Feart C, Mann G, Vina J, et al: Searching for an operational definition of frailty: a Delphi method based consensus statement: the frailty operative definitionconsensus conference project. J Gerontol A Biol Sci Med Sci 2013;68:62-67.

40 Rockwood K, Mitnitski A: Frailty in relation to the accumulation of deficits. J Gerontol A Biol Sci Med Sci 2007;62:722-727.

41 Rowe DA, Kemble CD, Robinson TS, Mahar MT: Daily walking in older adults: day-to-day variability and criterion-referenced validity of total daily step counts. J Phys Act Health 2007; 4:434-446.

42 Guimaraes RM, Isaacs B: Characteristics of the gait in old people who fall. Int Rehabil Med 1980;2:177-180.

43 Bridenbaugh SA, Kressig RW: Laboratory review: the role of gait analysis in seniors' mobility and fall prevention. Gerontology 2011; 57:256-264.

44 Schwenk M, Schmidt M, Pfisterer M, Oster P, Hauer K: Rollator use adversely impacts on assessment of gait and mobility during geriatric rehabilitation. J Rehabil Med 2011;43:424429.

45 Hardi I, Bridenbaugh SA, Gschwind YJ, Kressig RW: The effect of three different types of walking aids on spatio-temporal gait parameters in community-dwelling older adults. Aging Clin Exp Res 2014;26:221-228. 